\title{
Double outlet right ventricle with absent infundibular septum and common ventriculo-arterial valve
}

\author{
Paolo Ciliberti ${ }^{1}$, Aurelio Secinaro ${ }^{2}$, Gabriele Rinelli ${ }^{1}$, \\ Alessia Del Pasqua ${ }^{1}$, Adriano Carotti ${ }^{1}$ \\ ${ }^{1}$ Department of Pediatric Cardiology and Cardiac Surgery, \\ Bambino Gesù Children's Hospital IRCCS, Rome, Italy \\ ${ }^{2}$ Department of Imaging, Bambino Gesù Children's Hospital IRCCS, Rome, Italy
}

A 1-year-old male was referred to our unit because of double outlet right ventricle (Fig. 1A) with d-transposition of the great arteries and restrictive ventricular septal defect (VSD), located in subpulmonary position, but effectively committed to both pulmonary and aortic outflows because of absent infundibular septum (see Fig. 1, arrow). A first stage palliation had already been performed by atrial septectomy and pulmonary arterial banding. Preoperative cardiac magnetic resonance imaging showed complete absence of the infundibular septum (Fig. 1A, red asterisk) and fibrous continuity between the pulmonary and aortic valves (Fig. 1B). The tricuspid aortic valve was anterior to the right of the bicuspid pulmonary valve (Fig. 1C). The echocardiogram (Fig. 1D) also suspected partial
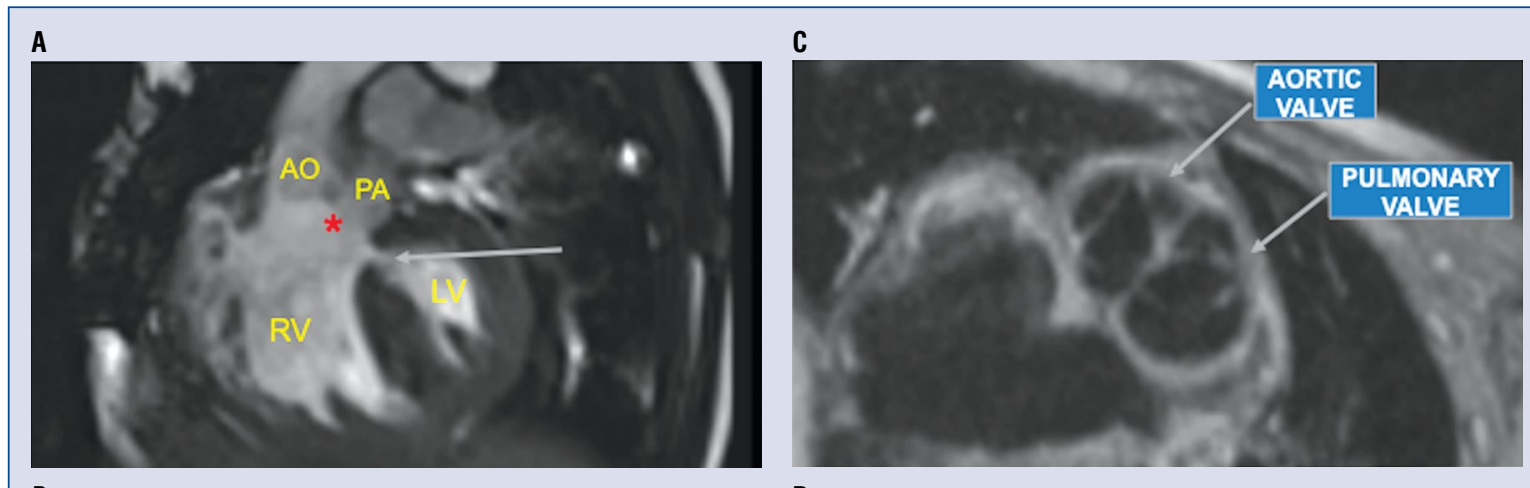

B

D
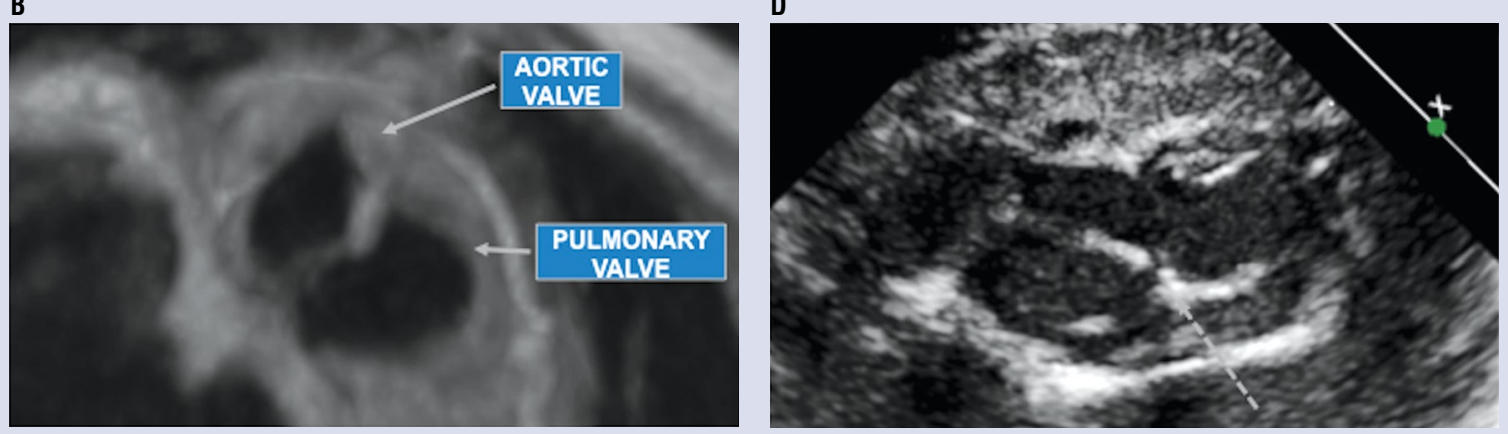

Figure 1. Cardiac magnetic resonance imaging (A, B, C) and echocardiographic (D) anatomical findings; $A O$ - aorta; LV — left ventricle; RV — right ventricle; PA — pulmonary artery.

Address for correspondence: Paolo Ciliberti, MD, PhD, Department of Pediatric Cardiology and Cardiac Surgery, Bambino Gesù Children's Hospital IRCCS, Piazza Sant'Onofrio 4, 00165, Rome, Italy, tel: (+39) 0668592796, fax: (+39) 0668594561, e-mail: paolo.ciliberti@opbg.net

Received: 10.01.2017 Accepted: 06.02.2017 
fusion of the two semilunar valves (Fig. 1D dotted arrow).

The patient underwent successful repair by pulmonary debanding, VSD enlargement, baffling of the left ventricle to the pulmonary valve and arterial switch operation. The surgical finding confirmed the presence of a common ventriculo-arterial annulus, with the VSD roofed by continuity of the pulmonary and aortic valve, and only a fibrous raphe interposing between the leaflets of the arterial valves.
A common ventriculo-arterial valve in patients with double outlet right ventricle and doubly committed VSD, was already described in autoptic series by Anderson et al. (Anderson RH, Baker EJ, Redington A, et al. Pediatric cardiology. Churchill Livingstone/Elsevier 2009: 842). This peculiar finding could be due to a deficient segregation of the distal part of the aortopulmonary septum, derived from the right and left endocardial cushions.

Conflict of interest: None declared 